\title{
Author Correction: Complete polarization control in multimode fibers with polarization and mode coupling
}

Wen Xiong, Chia Wei Hsu, Yaron Bromberg $\mathbb{0}$, Jose Enrique Antonio-Lopez, Rodrigo Amezcua Correa and Hui Cao

Correction to: Light: Science \& Application https://doi.org/10.1038/s41377-018-0047-4 published online 8 August 2018

We would like to correct an incomplete sentence in the last paragraph on page 3 .

"With a large number of modes in the fiber, we obtain." should be corrected to
"With a large number of modes in the fiber, we obtain PER >> 1."

We would like to apologize for any confusion this may have caused.

Published online: 29 April 2020 